
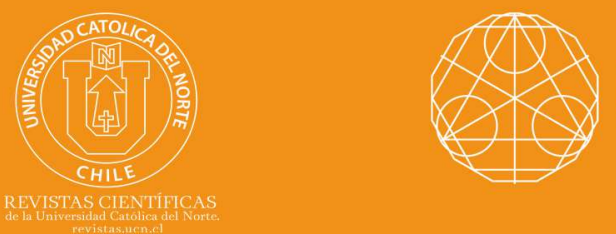

\title{
Stability, boundedness and existence of unique periodic solutions to a class of fourth order functional differential equations
}

Adeleke Timothy Ademola ${ }^{1}$ o orcid.org/0000-0002-1036-1681

${ }^{1}$ Obafemi Awolowo University, Department of Mathematics, Ile-Ife, Nigeria.

ఐatademola@oauife.edu.ng

\section{Abstract:}

In this paper a novel class of fourth order functional differential equations is discussed. By reducing the fourth order functional differential equation to system of first order, a suitable complete Lyapunov functional is constructed and employed to obtain sufficient conditions that guarantee existence of a unique periodic solution, asymptotic and uniform asymptotic stability of the zero solutions, uniform boundedness and uniform ultimate boundedness of solutions. The obtained results are new and include many prominent results in literature. Finally, two examples are given to show the feasibility and reliability of the theoretical results.

Keywords: Fourth order; Non linear functional differential equation; Uniform stability; Uniform ultimate boundedness; Periodic solutions.

MSC (2020): 34K12, 34K13, 34K20, 34K99.

\section{Cite this article as (IEEE citation style):}

A. T. Ademola, "Stability, boundedness and existence of unique periodic solutions to a class of fourth order functional differential equations", Proyecciones (Antofagasta, On line), vol. 40, no. 2, pp. 271-303, 2021, doi: 10.22199/issn.0717-6279-2021-02-0017

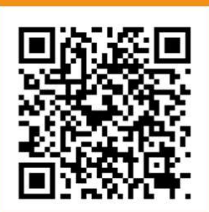

Article copyright: (C) 2021 Adeleke Timothy Ademola. This is an open access article distributed under the terms of the Creative Commons License, which permits unrestricted use and distribution provided the original author and source are credited. 


\section{Introduction}

The importance of functional differential equations(FDEs) in mathematical modelling cannot be over-emphasized, as it assumes a specified behaviour or phenomenon which depends on the present as well as the past state of a system. In other words, past events explicitly influence future results. Functional differential equation is the general name for a number of more specific types of differential equations such as delay differential equations, integro-differential equations, difference differential equations and so on, that are used in numerous applications in sciences and engineering technologies.

As a result of this, serious attention has been given by researchers to the study of qualitative behaviour of solutions to all branches of differential equations, (see for instance Ademola and Ogundiran [2], Burton [5, 6], Driver [8], Hale [9], Lakshmikantham et al. [14], Yoshizawa [23, 24] which contain basic background to the study. Other interesting papers on fourth order delay differential equations include but are not limited to Ademola [1], Adesina [3], Balamuralitharan [4], Cai and Meng [7], Kang and Si [10], Korkmaz and Tunç [11], Korkmaz [12, 13], Okoronkwo [15], Rahmane and Remili [16], Sadek [17], Sinha [18], Tejumola and Tchegnani [19], Tunç $[20,21,22])$. In this regard, these cited papers and their references contain outstanding results on the qualitative behaviour of solutions of fourth order differential equations with delay (constant or variables).

In 1989 Okoronkwo [15], used Razumikhin-type theorem to discuss the stability and boundedness of solutions for the fourth order delay differential equation

$\left.x^{(4)}+f\left(x^{\prime \prime}\right) x^{\prime \prime \prime}+\alpha_{2} x^{\prime \prime}+\beta_{2} x^{\prime \prime}(t-h)+g\left(x^{\prime}(t-h)\right)+\alpha_{4} x+\beta_{4} x(t-h)\right)=p(t)$,

where $\alpha_{2}, \beta_{2}, \alpha_{4}, \beta_{4}$ are constants and $h>0$ is a constant delay. The functions $f, g, p$ are completely continuous depending on the arguments displayed explicitly. In another interesting paper, employing the second method of Lyapunov, Sadek [17] studied sufficient conditions for the asymptotic stability of the zero solutions for the fourth order

$$
x^{(4)}+\alpha_{1} x^{\prime \prime \prime}+\alpha_{2} x^{\prime \prime}+\alpha_{3} x^{\prime}+f(x(t-r))=0
$$

and

$$
x^{(4)}+\alpha_{1} x^{\prime \prime \prime}+\alpha_{2} x^{\prime \prime}+\phi\left(x^{\prime}(t-r)\right)+f(x)=0,
$$


where $\alpha_{1}, \alpha_{2}, \alpha_{3}$ are constants, $r>0$ is a delay constant, $f$ and $\phi$ are continuous function such that $f(0)=0=\phi(0)$.

In another paper, Tunç [21] set-up criteria for asymptotic stability of the trivial solution for the fourth order delay differential equation

$$
x^{(4)}+\varphi\left(x^{\prime \prime}\right) x^{\prime \prime \prime}+h\left(x^{\prime}\right)\left(x^{\prime \prime}\right)+\phi\left(x^{\prime}(t-r)\right)+f(x(t-r))=0,
$$

where $r>0$ is a delay constant, $\varphi, h, \phi$ and $f$ are continuous functions with $\phi(0)=f(0)=0$. In [20] Tunç also considered a boundedness criterion for fourth order nonlinear ordinary differential equations with delay

$$
x^{(4)}+\varphi\left(x^{\prime \prime}\right) x^{\prime \prime \prime}+h\left(x^{\prime}(t-r)\right)+\phi\left(x^{\prime}(t-r)\right)+f(x(t-r))=P(\cdot),
$$

where $P(\cdot)=p\left(t, x, x^{\prime}, x^{\prime \prime}, x^{\prime \prime \prime}, x(t-r), x^{\prime}(t-r), x^{\prime \prime}(t-r)\right)$, the functions $\varphi, h, \phi, f$ and $p$ depend only on the variables displayed explicitly and $r$ is a positive constant fixed delay.

Balamuralitharan [4] employed the continuation theorem of Mawhin's coincidence degree theory to study the periodic solutions to the fourth order delay differential equation of the form

$$
x^{(4)}+a x^{\prime \prime \prime}+f\left(x^{\prime \prime}(t-r(t))\right)+g\left(x^{\prime}(t-r(t))\right)+h(x(t-r(t)))=p(t),
$$

where $a>0$ is a constant, $f, g, h$ and $p$ are real continuous functions defined on $\mathbf{R}=(-\infty, \infty)$ and $h$ is periodic with common period $\omega$. In 2017 Korkmaz and Tunç [11] gave sufficient conditions for the boundedness and square integrability of solutions of nonlinear fourth order differential equations with bounded delay

$$
\begin{gathered}
x^{(4)}+a(t)\left(g(x(t)) x^{\prime \prime}\right)^{\prime}+b(t)\left(q(x(t)) x^{\prime}(t)\right)^{\prime}+c(t) f(x(t)) x^{\prime}(t)+d(t) h(x(t-r(t))) \\
=p\left(t, x, x^{\prime}, x^{\prime \prime}, x^{\prime \prime \prime}\right),
\end{gathered}
$$

where $r$ is a bounded delay, $0 \leq r(t) \leq \psi, r^{\prime}(t) \leq \xi, 0<\xi<1$, $\xi$ and $\psi$ are positive constants, $a, b, c, d$ are continuous differentiable functions and $f, g, h, p, q$ are continuous functions depending only on the arguments shown.

Recently in [1], Ademola gave criteria for periodicity, stability and boundedness of solutions to fourth order delay differential equation

$x^{(4)}+\varphi\left(x, x^{\prime}, x^{\prime \prime}, x^{\prime \prime \prime}\right) x^{\prime \prime \prime}+f\left(x^{\prime}(t-r), x^{\prime \prime}(t-r)\right)+g\left(x(t-r), x^{\prime}(t-r)\right)+h(x(t-r))=p(\cdot)$, 
where $p(\cdot)=p\left(t, x, x^{\prime}, x^{\prime \prime}, x^{\prime \prime \prime}, x(t-r), x^{\prime}(t-r), x^{\prime \prime}(t-r)\right), r>0$ is a delay constant, $\varphi, f, g, h$ and $p$ are continuous functions in their respective arguments. Observations from relevant literature shows that works on fourth order differential equations (or fourth order with bounded delay) are extremely scared. The objective of this paper is to study stability, uniform asymptotic stability of the zero solution, boundedness, uniform ultimate boundedness and existence of a unique $\omega$-periodic solution for the fourth order delay differential equation

$$
\begin{aligned}
x^{(4)}(t) & +\phi\left(t, x(t), x^{\prime}(t), x^{\prime \prime}(t), x^{\prime \prime \prime}(t)\right) x^{\prime \prime \prime}(t)+f\left(x(t-r(t)), x^{\prime}(t-r(t)), x^{\prime \prime}(t-r(t))\right) \\
& +g\left(x(t-r(t)), x^{\prime}(t-r(t))\right)+h(x(t-r(t)))=P^{*}(\cdot),
\end{aligned}
$$

where $P^{*}(\cdot):=p\left(t, x(t), x^{\prime}(t), x^{\prime \prime}(t), x^{\prime \prime \prime}(t), x(t-r(t)), x^{\prime}(t-r(t)), x^{\prime \prime}(t-\right.$ $r(t)))$. Equation 1.1 is equivalent to system of first order differential equations

$$
\begin{aligned}
& x^{\prime}(t)=y(t), y^{\prime}(t)=z(t), z^{\prime}(t)=u(t) \\
& u^{\prime}(t)=P(\cdot)-\phi(t, x, y, z, u) u-f(x, y, z)-g(x, y)-h(x)+\int_{t-r(t)}^{t} h^{\prime}(x(s)) y(s) d s \\
& +\int_{t-r(t)}^{t}\left[g_{x}(x(s), y(s)) y(s)+g_{y}(x(s), y(s)) z(s)+f_{x}(x(s), y(s), z(s)) y(s)\right] d s \\
& +\int_{t-r(t)}^{t}\left[f_{y}(x(s), y(s), z(s)) z(s)+f_{z}(x(s), y(s), z(s)) u(s)\right] d s,
\end{aligned}
$$

where $P(\cdot)=p(t, x, y, z, u, x(t-r(t), y(t-r(t), z(t-r(t)))$, the functions $\phi, f, g, h$ and $p$ are continuous in their respective arguments on $\mathbf{R}^{+} \times \mathbf{R}^{4}, \mathbf{R}^{3}$, $\mathbf{R}^{2}, \mathbf{R}$ and $\mathbf{R}^{+} \times \mathbf{R}^{7}$ respectively with $\mathbf{R}^{+}=[0, \infty), \mathbf{R}=(-\infty, \infty)$ and $0 \leq$ $r(t) \leq \gamma, \gamma>0$ being a constant whose value will be established later. The primes as usual, stand for differentiation with respect to the independent variable $t$ and the derivatives $h^{\prime}(x), g_{x}(x, y), g_{y}(x, y), f_{x}(x, y, z), f_{y}(x, y, z)$ and $f_{z}(x, y, z)$ exist and are continuous for all $x, y, z$. Continuity of these functions is sufficient for the existence of the solutions of equation 1.1. Furthermore, it is assumed that the functions $\phi, f, g$ and $p$ in the equation 1.1 satisfy a Lipschitz condition in their respective arguments.

This work is motivated by the recent works in $[1,4,7,11,12]$ and [20]. The obtained results are new and include many existing results in the literature. Despite the attention that has been given to the fourth order nonlinear differential equations with delay, there are no situation where the nonlinear function $\phi$ depends explicitly on the independent variable $t$, functions $f, g, h$ and $p$ simultaneously depend on $(t-r(t))$. Besides, a suitable complete Lyapunov functional is employed in this investigation compare 
with the existing results where an incomplete Lyapunov functional are employed. These are our principal contributions. The next section gives basic preliminary results used in this work. The main results of this paper are stated and proved in Sections 3 and 4, while examples and discussions are given in Section 5.

\section{Preliminary Results}

Consider the following general nonlinear non-autonomous delay differential equation

$$
\dot{X}=\frac{d X}{d t}=F\left(t, X_{t}\right), X_{t}=X(t+\theta),-r \leq \theta<0, t \geq 0,
$$

where $F: \mathbf{R}^{+} \times C_{H} \rightarrow \mathbf{R}^{n}$ is a continuous mapping, $F(t+\omega, \phi)=F(t, \phi)$ for all $\phi \in C$ and for some positive constant $\omega$. We assume that $F$ takes closed bounded sets into bounded sets in $\mathbf{R}^{n}$. $(C,\|\cdot\|)$ is the Banach space of continuous function $\varphi:[-r, 0] \rightarrow \mathbf{R}^{n}$ with supremum norm, $r>0$; for $H>0$, we define $C_{H} \subset C$ by $C_{H}=\{\varphi \in C:\|\varphi\|<H,\} C_{H}$ is the open $H$-ball in $C, C=C\left([-r, 0], \mathbf{R}^{n}\right)$.

Definition 2.1. (See [9]). Suppose that $F(t, 0)=0$ for all $t \in \mathbf{R}$. The solution $X_{t}=0$ is said to be stable for any $t_{0} \in \mathbf{R}, \epsilon>0$, there is a $\delta=\delta\left(\epsilon, t_{0}\right)$ such that $\|\phi\|_{r}<\delta$ implies $\left\|X_{t}\left(t ; t_{0}, \phi\right)\right\|<\epsilon$ for $t \geq t_{0}-r$. The solution $X_{t}=0$ of 2.1 is uniformly stable on $\left(t_{0}, \infty\right)$ if it is stable at each $t_{0}$ and the number $\delta$ is independent of $t_{0}$ (i.e $\delta(\epsilon)$ depends only on $\epsilon$ ).

Definition 2.2. (See [9]). The solution $X_{t}=0$ of 2.1 is said to be asymptotically stable at $t_{0}$ if it is stable at $t_{0}$ then there exists number $\delta_{1}=$ $\delta_{1}\left(t_{0}\right)>0$ such that whenever $\|\phi\|_{r}<\delta_{1}$,

$$
\lim _{t \rightarrow \infty} X_{t}\left(t ; t_{0}, \phi\right)=0 .
$$

The solution $X_{t}=0$ of 2.1 is uniformly asymptotically stable if it is uniformly stable and furthermore, there exits $\delta_{1}>0$ (independent of $t_{0}$ ) such that for each $t_{0}$ and $\|\phi\|_{r}<\delta_{1}$,

$$
X_{t}\left(t ; t_{0}, \phi\right) \rightarrow 0
$$

as $t \rightarrow \infty$. 
Definition 2.3. (See [14]). A solution of 2.1 is bounded if there is an $M=M\left(t_{0}, \phi\right)>0$ such that

$$
\left\|X_{t}\left(t ; t_{0}, \phi\right)\right\|<M
$$

for all $t \geq t_{0}-r$.

Definition 2.4. (See [14]). The solutions of 2.1 are equi-bounded if for any $H>0$ and $t_{0} \in \mathbf{R}$ there exists an $M=M\left(t_{0}, H\right)>0$ such that if $\|\phi\|<H$, then

$$
\left\|X_{t}\left(t ; t_{0}, \phi\right)\right\|<M
$$

for all $t \geq t_{0}$.

Definition 2.5. (See [14]). The solutions of 2.1 are uniformly bounded if the $M$ in Definition ?? is independent of $t_{0}$.

Definition 2.6. (See [14]). The solutions of 2.1 are ultimately bounded for bound $M$, if there exist an $M>0$ and a $T=T\left(t_{0}, \phi\right)>0$ such that for every solution $X_{t}\left(t_{0}, \phi\right)$ of 2.1

$$
\left\|X_{t}\left(t ; t_{0}, \phi\right)\right\|<M
$$

for all $t \geq t_{0}+T$.

Definition 2.7. (See [14]). The solutions of 2.1 are equi-ultimately bounded for bound $M$, if there exist an $M>0$ and if corresponding to any $H>0$ and $t_{0} \in \mathbf{R}$ there exists a $T=T\left(t_{0}, H\right)>0$ such that $\|\phi\|<H$ implies that

$$
\left\|X_{t}\left(t ; t_{0}, \phi\right)\right\|<M
$$

for all $t \geq t_{0}+T$.

Definition 2.8. (See [14]). The solutions of 2.1 are uniformly ultimately bounded for bound $M$, if the $T$ in Definition 2.7 is independent of $t_{0}$.

Definition 2.9. (See [5]). A continuous function $W: \mathbf{R}^{+} \rightarrow \mathbf{R}^{+}$with $W(0)=0, W(s)>0$ if $s \neq 0$, and $W$ strictly increasing is a wedge. (We denote wedges by $W$ or $W_{i}$, where $i$ is an integer).

Lemma 2.10. (See [24]). Suppose that $F(t, \phi) \in \bar{C}_{0}(\phi)$ and $F(t, \phi)$ is periodic in $t$ of period $\omega, \omega \geq r$ and consequently for any $\alpha>0$ there exists an $L(\alpha)>0$ such that $\phi \in C_{\alpha}$ implies $|F(t, \phi)| \leq L(\alpha)$. Suppose that a continuous Lyapunov functional $V(t, \phi)$ exists, defined on $t \in \mathbf{R}^{+}, \phi \in S^{*}$, $S^{*}$ is the set of $\phi \in C$ such that with $|\phi(0)| \geq H$ ( $H$ may be large) and that $V(t, \phi)$ satisfies the following conditions: 
(i) $a(|\phi(0)|) \leq V(t, \phi) \leq b(\|\phi\|)$, where $a(r)$ and $b(r)$ are continuous, increasing and positive for $r \geq H$ and $a(r) \rightarrow \infty$ as $r \rightarrow \infty$;

(ii) $\dot{V}_{2.1}(t, \phi) \leq-c(|\phi(0)|)$, where $c(r)$ is continuous and positive for $r \geq$ $H$.

Suppose that there exists an $H_{1}>0, H_{1}>H$, such that

$$
h L\left(\gamma^{*}\right)<H_{1}-H,
$$

where $\gamma^{*}>0$ is a constant which is determined in the following way: By the condition on $V(t, \phi)$ there exist $\alpha>0, \beta>0$ and $\gamma>0$ such that $b\left(H_{1}\right) \leq a(\alpha), b(\alpha) \leq a(\beta)$ and $b(\beta) \leq a(\gamma) \cdot \gamma^{*}$ is defined by $b(\gamma) \leq a\left(\gamma^{*}\right)$. Under the above conditions, there exists a periodic solution of 2.1 of period $\omega$. In particular, the relation 2.2 can always be satisfied if $h$ is sufficiently small.

Lemma 2.11. (See [24]). Suppose that $F(t, \phi)$ is defined and continuous on $0 \leq t \leq c, \phi \in C_{H}$ and that there exists a continuous Lyapunov functional $V(t, \phi, \varphi)$ defined on $0 \leq t \leq c, \phi, \varphi \in C_{H}$ which satisfy the following conditions:

(i) $V(t, \phi, \varphi)=0$ if $\phi=\varphi$;

(ii) $V(t, \phi, \varphi)>0$ if $\phi \neq \varphi$;

(iii) for the associated system

$$
\dot{x}(t)=F\left(t, x_{t}\right), \quad \dot{y}(t)=F\left(t, y_{t}\right)
$$

we have $V_{2.3}^{\prime}(t, \phi, \varphi) \leq 0$, where for $\|\phi\|=H$ or $\|\varphi\|=H$, we understand that the condition $V_{2.3}^{\prime}(t, \phi, \varphi) \leq 0$ is satisfied in the case $V^{\prime}$ can be defined.

Then, for given initial value $\phi \in C_{H_{1}}, H_{1}<H$, there exists a unique solution of 2.1 .

Lemma 2.12. (See [24]). Suppose that a continuous Lyapunov functional $V(t, \phi)$ exists, defined on $t \in \mathbf{R}^{+},\|\phi\|<H, 0<H_{1}<H$ which satisfies the following conditions:

(i) $a(\|\phi\|) \leq V(t, \phi) \leq b(\|\phi\|)$, where $a(r)$ and $b(r)$ are continuous, increasing and positive, 
(ii) $\dot{V}_{2.1}(t, \phi) \leq-c(\|\phi\|)$, where $c(r)$ is continuous and positive for $r \geq 0$, then the zero solution of 2.1 is uniformly asymptotically stable.

Lemma 2.13. (See [5]). Let $V: \mathbf{R}^{+} \times C \rightarrow \mathbf{R}$ be continuous and locally Lipschitz in $\phi$. If

(i) $W_{0}\left(\left|X_{t}\right|\right) \leq V\left(t, X_{t}\right) \leq W_{1}\left(\left|X_{t}\right|\right)+W_{2}\left(\int_{t-r(t)}^{t} W_{3}\left(X_{t}(s)\right) d s\right)$ and

(ii) $\dot{V}_{2.1}\left(t, X_{t}\right) \leq-W_{4}\left(\left|X_{t}\right|\right)+N$, for some $N>0$ where $W_{i}(i=$ $0,1,2,3,4)$ are wedges.

Then $X_{t}$ of 2.1 is uniformly bounded and uniformly ultimately bounded for bound $M$.

\section{Stability Results}

This section state and prove results on stability and uniform asymptotic stability of zero solution for the fourth order delay differential equation

$$
\begin{aligned}
x^{(4)}(t) & +\phi\left(t, x(t), x^{\prime}(t), x^{\prime \prime}(t), x^{\prime \prime \prime}(t)\right) x^{\prime \prime \prime}(t)+f\left(x(t-r(t)), x^{\prime}(t-r(t)), x^{\prime \prime}(t-r(t))\right) \\
& +g\left(x(t-r(t)), x^{\prime}(t-r(t))\right)+h(x(t-r(t)))=0,
\end{aligned}
$$

where $\phi, f, g$ and $h$ are the functions defined in Section 1. As usual, equation 3.1 in its equivalent system of first order is

$$
\begin{aligned}
& x^{\prime}(t)=y(t), y^{\prime}(t)=z(t), z^{\prime}(t)=u(t), \\
& u^{\prime}(t)=-\phi(t, x, y, z, u) u-f(x, y, z)-g(x, y)-h(x)+\int_{t-r(t)}^{t}\left[f_{x}(x(s), y(s), z(s)) y(s)\right] d s \\
& +\int_{t-r(t)}^{t}\left[f_{y}(x(s), y(s), z(s)) z(s)+f_{z}(x(s), y(s), z(s)) u(s)+g_{x}(x(s), y(s)) y(s)\right] d s \\
& +\int_{t-r(t)}^{t}\left[g_{y}(x(s), y(s)) z(s)+h^{\prime}(x(s)) y(s)\right] d s .
\end{aligned}
$$

Next, the continuously differentiable functional $V(X)=V\left(x_{t}, y_{t}, z_{t}, u_{t}\right)$ employed in this paper is defined as

$$
\begin{aligned}
& 2 V(X)=2 \alpha_{2} \int_{0}^{x} h(s) d s+2 \int_{0}^{y} g(x, s) d s+a^{2} x^{2}+\left(b \alpha_{2}-d \alpha_{1}+b^{2}\right) y^{2} \\
& +\left(a+b \alpha_{1}-\alpha_{1}+c^{2}\right) z^{2}+\left(1+\alpha_{1}\right) u^{2}+2 y h(x)+2 \alpha_{2} z h(x)+2\left(a \alpha_{2}+c \alpha_{1}+b c\right) y z \\
& +2\left(\alpha_{2}+b\right) y u+2(1+c) z u+2 a b x y+2 a c x z+2 a x u \\
& +2 \int_{-r(t)}^{0} \int_{t+s}^{t}\left(\mu_{1} y^{2}(\theta)+\mu_{2} z^{2}(\theta)+\mu_{3} u^{2}(\theta)\right) d \theta d s,
\end{aligned}
$$

where 


$$
\alpha_{1}:=\epsilon+\frac{1}{a}, \alpha_{2}:=\epsilon+\frac{d}{c},
$$

are positive constants, $\epsilon$ and $\mu_{i}(i=1,2,3)$ are constants that will be determined later. The following stability results are presented.

Theorem 3.1. In addition to the basic conditions on the non linear functions $\phi, f, g$ and $h$, suppose that $a, b, c, d, \alpha_{1}, \alpha_{2}, \beta, \delta, \delta_{0}, \gamma$ and $M_{i}(i=$ $1,2, \ldots, 6)$ are positive constants such that

(i) $a \leq \phi(t, x, y, z, u)$ for all $t \geq 0, x, y, z, u$;

(ii) $b \leq \frac{f(x, y, z)}{z}$, for all $x, y, z \neq 0,\left|f_{x}(\cdot)\right| \leq M_{1},\left|f_{y}(\cdot)\right| \leq M_{2},\left|f_{z}(\cdot)\right| \leq M_{3}$, for all $x, y, z$;

(iii) $c \leq \frac{g \mid(x, y)}{y}$, for all $x, y \neq 0,\left|g_{x}(x, y)\right| \leq M_{4},\left|g_{y}(x, y)\right| \leq M_{5}$, for all $x, y$;

(iv) $h(0)=0, \delta_{0} \leq \frac{h(x)}{x}$ for all $x \neq 0, h^{\prime}(x) \leq d,\left|h^{\prime}(x)\right| \leq M_{6}$, for all $x$;

(v) $c<a b, \delta \leq a b c-c^{2}-a^{2} d$; and

(vi) $0 \leq r(t) \leq \gamma, r^{\prime}(t) \leq \beta, 0<\beta<1$.

Then the zero solution of system 3.2 is asymptotically stable provided that

$$
\gamma<\min \left\{\frac{\delta_{0}}{A_{1}}, \frac{b(a+c)(1-\beta)}{A_{2}}, \frac{\delta(1-\beta)}{A_{3}}, \frac{(a+c)(1-\beta)}{A_{4}}\right\},
$$

where:

$$
\begin{gathered}
A_{1}:=2 \sum_{i=1}^{6} M_{i} ; \\
A_{2}:=2\left[\left(b+\alpha_{2}\right)(1-\beta) \sum_{i=1}^{6} M_{i}+\left(M_{1}+M_{4}+M_{6}\right)\left(a+b+c+\frac{1}{a c}(c+a d+2 a \epsilon)+2\right)\right] ; \\
A_{3}:=4 a c\left[(1+c)(1-\beta) \sum_{i=1}^{6} M_{i}+\left(M_{2}+M_{5}\right)\left(a+b+c+\frac{1}{a c}(c+a d+2 a \epsilon)+2\right)\right] ; \\
A_{4}:=2\left[\left(1+\alpha_{1}\right)(1-\beta) \sum_{i=1}^{6} M_{i}+M_{3}\left(a+b+c+\frac{1}{a c}(c+a d+2 a \epsilon)+2\right)\right] ; \\
\frac{\delta_{0}}{2 a c \alpha_{0}} \leq \epsilon \leq \min \left\{\frac{2 a b}{c}, \frac{2 c}{a}\right\} ;
\end{gathered}
$$

and

$$
\alpha_{0}:=a b+b c d^{-1}>0
$$


Theorem 3.2. If assumptions (i), (ii), (v) and (vi) of Theorem 3.1 hold and in addition to assumptions (iii) and (iv) there exist positive constants $c_{1}$ and $d_{1}$ such that

(iii) $c \leq \frac{g(x, y)}{y} \leq c_{1}$ for all $x, y \neq 0$; and

(iv) $\delta_{0} \leq \frac{h(x)}{x} \leq d_{1}$ for all $x \neq 0$.

Then the zero solution of system 3.2 is uniformly asymptotically stable provided that the inequality 3.5 a holds.

Remark 3.1. Proper scrutiny of equation 3.1, system 3.2, equations 3.3, 3.4, Theorems 3.1, 3.2, the existing relevant equations and results on fourth order functional differential equations, the following observations are recorded:

(i) If $\phi\left(t, x, x^{\prime}, x^{\prime \prime}, x^{\prime \prime \prime}\right)=a, f\left(x(t-r(t)), x^{\prime}(t-r(t)), x^{\prime \prime}(t-r(t))\right)=b x^{\prime \prime}$ $g\left(x(t-r(t)), x^{\prime}(t-r(t))\right)=c x^{\prime}$ and $h(x(t-r(t)))=d x$, then equation 3.1 reduces to a linear fourth order differential equation

$$
x^{(4)}(t)+a x^{\prime \prime \prime}(t)+b x^{\prime \prime}(t)+c x^{\prime}(t)+d x(t)=0
$$

and conditions (i) to (vi) of Theorems 3.1 and 3.2 specialize to the well-known Routh-Hurtwitz condition for asymptotic stability of the zero solution of equation 3.6, that is $a>0, b>0, a b-c>0, c>0$, $a b c-c^{2}-a^{2} d>0$ and $d>0$;

(ii) Whenever $\phi\left(t, x, x^{\prime}, x^{\prime \prime}, x^{\prime \prime \prime}\right)=f\left(x^{\prime \prime}\right), f\left(x(t-r(t)), x^{\prime}(t-r(t)), x^{\prime \prime}(t-\right.$ $r(t)))=\alpha_{2} x^{\prime \prime}+\beta_{2} x^{\prime \prime}(t-h), g\left(x(t-r(t)), x^{\prime}(t-r(t))\right)=g\left(x^{\prime}(t-h)\right)$, $h(x(t-r(t)))=\alpha_{4} x+\beta_{4} x(t-h), r(t)=h$ where $\alpha_{2}, \beta_{2}, \alpha_{4}, \beta_{4}$ and $h$ are constants, then equation 3.1 reduces to that discussed in [15]. Razumikhin-type approach was introduced to obtain stability result in [15], thus our hypotheses are dissimilar;

(iii) The case when $\phi\left(t, x, x^{\prime}, x^{\prime \prime}, x^{\prime \prime \prime}\right)=\alpha_{1}, f\left(x(t-r(t)), x^{\prime}(t-r(t)), x^{\prime \prime}(t-\right.$ $r(t)))=\alpha_{2} x^{\prime \prime}, g\left(x(t-r(t)), x^{\prime}(t-r(t))\right)=\alpha_{3} x^{\prime}$ or $\phi\left(x^{\prime}(t-r)\right), h(x(t-$ $r(t)))=f(x(t-r))$ or $f(x)$ and $r(t)=r$, where $\alpha_{1}, \alpha_{2}, \alpha_{3}$ and $r$ are positive constants, then equation 3.1 is narrowed down to the case discussed [17]. Some of our hypotheses are similar to that of [17];

(iv) Whenever $\phi(\cdot)=\varphi\left(x^{\prime \prime}\right), f(\cdot)=h\left(x^{\prime}\right) x^{\prime \prime}, g\left(x, x^{\prime}\right)=\phi\left(x^{\prime}(t-r)\right), h(x(t-$ $r(t)))=h(x(t-r))$ and $r(t)=r$ where $r>0$ is a constant delay, then equation 3.1 is pined down to the case discussed in [21], hence the results of Theorem 3.2 include and extends the stability result obtained in [21]; 
(v) Also, the stability results obtained in $[1,7,10,18]$ are special case of the results of this paper; and

(vi) The main tools (the Lyapunov functional) employed in $[1,7,15,17$, 21] are incomplete functionals compare with equation 3.3 , a suitable complete functional, which can replace those used in $[1,7,15,17,21]$.

Next, two major lemmas that are crucial to the proves of our results, are as follow.

Lemma 3.3. In view of the assumptions of Theorem 3.2 there exist positive constants $D_{1}=D_{1}\left(a, b, c, d, \delta, \delta_{0}\right)$ and $D_{2}=D_{2}\left(a, b, c, d, c_{1}, d_{1}, \gamma, \mu_{1}, \mu_{2}, \mu_{3}\right)$ such that

$$
D_{1}\left(x^{2}+y^{2}+z^{2}+u^{2}\right) \leq V\left(X_{t}\right) \leq D_{2}\left(x^{2}+y^{2}+z^{2}+u^{2}\right)
$$

for all $t \geq 0, x, y, z$ and $u$.

Proof. Let $\left(X_{t}\right)=\left(x_{t}, y_{t}, z_{t}, u_{t}\right)$ be any solution of equation 3.2. Clearly the function $V$ defined by 3.3 satisfies

$$
V(\mathbf{0})=0,
$$

where $(\mathbf{0})=(0,0,0,0)$. Furthermore, equation 3.3 can be re-written in the form

$$
\begin{aligned}
& 2 V\left(X_{t}\right)=2 \alpha_{2} \int_{0}^{x} h(s) d s-\frac{1}{c} h^{2}(x)+2 \int_{0}^{y} g(x, s) d s-c y^{2}+\left(b \alpha_{2}-d \alpha_{1}-a \alpha_{2}^{2}\right) y^{2} \\
& +\left(b \alpha_{1}-\alpha_{2}-c \alpha_{1}^{2}\right) z^{2}+\left(\alpha_{1}-\frac{1}{a}\right) u^{2}+\frac{1}{a}\left(a \alpha_{2} y+a z+u\right)^{2}+\frac{1}{c}\left(h(x)+c y+c \alpha_{1} z\right)^{2} \\
& +(a x+b y+c z+u)^{2}+2 \int_{-r(t)}^{0} \int_{t+s}^{t}\left(\mu_{1} y^{2}(\theta)+\mu_{2} z^{2}(\theta)+\mu_{3} u^{2}(\theta)\right) d \theta d s .
\end{aligned}
$$

Now, the first two terms of equation 3.8 is

$$
2 \alpha_{2} \int_{0}^{x} h(s) d s-\frac{1}{c} h^{2}(x) \geq \frac{1}{c}\left(c \alpha_{2}-d\right) \delta_{0} x^{2}=\epsilon \delta_{0} x^{2}
$$

for all $x$. Also, assumption (iii) of Theorem 3.1 implies that

$$
2 \int_{0}^{y} g(x, s) d s-c y^{2}=2 \int_{0}^{y}\left(\frac{g(x, s)}{s}-c\right) s d s \geq 0
$$


for all y. Equation 3.4 and assumption (v ) of Theorem 3.1 yields

$$
b-\alpha_{2}-c \alpha_{1}=\frac{1}{a c}\left(a b c-c^{2}-a^{2} d\right)-\epsilon(a+c) \geq \frac{\delta}{a c}-\epsilon(a+c) .
$$

Note that $a b c>a b c-c^{2}>a^{2} d$ implies that $a<b c d^{-1}$ and $c<a b$ so that

$$
b-\alpha_{2}-c \alpha_{1} \geq \frac{\delta}{a c}-\epsilon\left(b c d^{-1}+a b\right)=\frac{\delta}{a c}-\epsilon \alpha_{0},
$$

Equation 3.4, estimate 3.11 and the fact that $c \alpha_{1} \epsilon>0$ gives the coefficient of $y^{2}$ in equation 3.8 as

$$
\begin{aligned}
b \alpha_{2}-d \alpha_{1}-a \alpha_{2}^{2} & =\alpha_{2}\left(b-a \alpha_{2}-c \alpha_{1}\right)+\alpha_{1}\left(c \alpha_{2}-d\right) \\
& \geq \alpha_{2}\left(\frac{\delta}{a c}-\epsilon \alpha_{0}\right)+\alpha_{1}\left(c \alpha_{2}-d\right) \\
& =\alpha_{2}\left(\frac{\delta}{a c}-\epsilon \alpha_{0}\right)+c \epsilon \alpha_{1} \\
& \geq \alpha_{2}\left(\frac{\delta}{a c}-\epsilon \alpha_{0}\right) .
\end{aligned}
$$

Now, define $\epsilon=\frac{\delta}{2 a c \alpha_{0}}$ so that

$$
b \alpha_{2}-d \alpha_{1}-a \alpha_{2}^{2} \geq \frac{\delta}{4 a^{2} c \alpha_{0}}\left(\delta+2 a d \alpha_{0}\right) .
$$

The coefficient of $z^{2}$ in 3.8 becomes

$$
b \alpha_{1}-\alpha_{2}-c \alpha_{1}^{2}=\alpha_{1}\left(b-a \alpha_{2}-c \alpha_{1}\right)+\alpha_{2}\left(a \alpha_{2}-1\right) .
$$

Using equation 3.4 and estimate 3.11 , to obtain

$$
b \alpha_{1}-\alpha_{2}-c \alpha_{1}^{2} \geq \alpha_{1}\left(\frac{\delta}{a c}-\epsilon \alpha_{0}\right)=\frac{\delta}{4 a^{2} c^{2} \alpha_{0}}\left(\delta+2 c \alpha_{0}\right) .
$$

Further, the coefficient of $u^{2}$ is

$$
\left(\alpha_{1}-\frac{1}{a}\right)=\epsilon=\frac{\delta}{2 a c \alpha_{0}}
$$

Moreover, since $a>0$ and $c>0$ it follows that

$$
\left.\frac{1}{a} a \alpha_{2} y+a z+u\right)^{2} \geq 0, \frac{1}{c}\left(h(x)+c y+c \alpha_{1} z\right)^{2} \geq 0,(a x+b y+c z+u)^{2} \geq 0
$$


for all $x, y, z$ and $u$. Finally, since the double integrals in 3.8 is non-negative, estimates $3.9,3.10,3.12,3.13,3.14$ and 3.15 give rise to the existence of a positive constant $\beta_{1}=\beta_{1}\left(a, b, c, d, \delta, \delta_{0}\right)$ such that

$$
V\left(X_{t}\right) \geq \beta_{1}\left(x^{2}+y^{2}+z^{2}+u^{2}\right)
$$

for all $t \geq 0, x, y, z, u$, where

$$
\beta_{1}:=\frac{1}{2} \min \left\{\frac{\delta \delta_{0}}{2 a c \alpha_{0}}, \frac{\delta\left(\delta+2 a d \alpha_{0}\right)}{4 a^{2} c \alpha_{0}}, \frac{\delta\left(\delta+2 c \alpha_{0}\right)}{4 a^{2} c^{2} \alpha_{0}}, \frac{\delta}{2 a c \alpha_{0}}\right\} .
$$

Inequality 3.16 shows that the functional $V\left(X_{t}\right)$ defined by 3.3 is positive semi-definite. Moreover, inequality 3.16 gives the following

$$
V\left(X_{t}\right)=0 \text { if and only if } x^{2}+y^{2}+z^{2}+u^{2}=0,
$$

and

$$
V\left(X_{t}\right)>0 \text { if and only if } x^{2}+y^{2}+z^{2}+u^{2} \neq 0 .
$$

Estimates 3.17 and 3.18 results to

$$
V\left(X_{t}\right) \rightarrow+\infty \operatorname{as} x^{2}+y^{2}+z^{2}+u^{2} \rightarrow \infty .
$$

Estimate 3.19 shows that the functional $V\left(X_{t}\right)$ is radially unbounded. Furthermore, the obvious inequality $2 x y \leq x^{2}+y^{2}$, assumptions (iii)', (vi) ${ }^{\prime}$ of Theorem 3.2, and the fact that $r(t) \leq \gamma$ for all $t \geq 0$, equation 3.3 becomes

$$
V\left(X_{t}\right) \leq \beta_{2}\left(x^{2}+y^{2}+z^{2}+u^{2}\right)
$$

for all $t \geq 0, x, y, z, u$, where

$$
\begin{aligned}
\beta_{2}:= & \frac{1}{2} \max \left\{a(a+b+c+1)+\left(\alpha_{1}+\alpha_{2}+1\right) d, \alpha_{1}(c+d)+\alpha_{2}(a+b+1)+b(a+b+c+1)\right. \\
& +\gamma^{2} \mu_{1}+c_{1}+d_{1}, \alpha_{1}\left(b+c+d_{1}\right)+\alpha_{2}(1+a)+c(a+b+c+1)+\gamma^{2} \mu_{2}+a+1, \\
& \left.\alpha_{1}+\alpha_{2}+a+b+c+\gamma^{2} \mu_{3}\right\} .
\end{aligned}
$$

Inequality 3.20 unveils that the functional $V\left(X_{t}\right)$ of 3.3 is decrescent. Estimates 3.16 and 3.20 established estimate 3.7 with $\beta_{1}$ and $\beta_{2}$ equivalent to $D_{1}$ and $D_{2}$ respectively. This completes the prove of Lemma 3.3. 
Lemma 3.4. If the assumptions of Theorem 3.1 hold, then there exists a positive constant $D_{3}=D_{3}\left(a, b, c, \alpha_{1}, \alpha_{2}, \beta, \gamma, M_{i}\right), i=1,2, \cdots, 6$ such that along a solution of system 3.2

$$
V_{3.2}^{\prime}\left(X_{t}\right) \leq-D_{3}\left(x^{2}+y^{2}+z^{2}+u^{2}\right)
$$

for all $t \geq 0, x, y, z$ and $u$.

Proof. Let $\left(X_{t}\right)$ be any solution of system 3.2, the first partial derivative of functional $V\left(X_{t}\right)$ with respect to the independent variable $t$ along a solution of system 3.2 is

$$
\begin{aligned}
& V_{3.2}^{\prime}\left(X_{t}\right)=-a \frac{h(x)}{x} x^{2}-\left[\left(\alpha_{2}+b\right) \frac{g(x, y)}{y}-\left(a b+h^{\prime}(x)\right)\right] y^{2} \\
& -\left[(1+c) \frac{f(\cdot)}{z}-\left(a \alpha_{2}+\left(\alpha_{1}+b\right) c\right)\right] z^{2}-\left[\left(1+\alpha_{1}\right) \phi(\cdot)-(1+c)\right] u^{2} \\
& -\left[\left(b \frac{h(x)}{x}+a \frac{g(x, y)}{y}\right)-a^{2}\right] x y-\left[\left(c \frac{h(x)}{x}+a \frac{f(\cdot)}{z}\right)-a b\right] x z \\
& -\left[\left(\frac{h(x)}{x}+a \phi(\cdot)\right)-a c\right] x u-\left[\left(c \frac{g(x, y)}{y}+\left(b+\alpha_{2}\right) \frac{f(\cdot)}{z}\right)\right. \\
& \left.-\left(\alpha_{1} h^{\prime}(x)+a c+b \alpha_{2}-d \alpha_{1}+b^{2}\right)\right] y z \\
& -\left[\left(\left(1+\alpha_{1}\right) \frac{g(x, y)}{y}+\left(b+\alpha_{2}\right) \phi(\cdot)\right)-\left(a\left(1+\alpha_{2}\right)+\left(\alpha_{1}+b\right) c\right)\right] y u \\
& -\left[\left(\left(1+\alpha_{1}\right) \frac{f(\cdot)}{z}+(1+c) \phi(\cdot)\right)-\left(a+b\left(1+\alpha_{1}\right)+c^{2}\right)\right] z u \\
& -\left(1-r^{\prime}(t)\right) \int_{t-r(t)}^{t}\left[\mu_{1} y^{2}(s)+\mu_{2} z^{2}(s)+\mu_{3} u^{2}(s)\right] d s \\
& +y \int_{0}^{y} g_{x}(x, s) d s+r(t)\left[\mu_{1} y^{2}+\mu_{2} z^{2}+\mu_{3} u^{2}\right] \\
& +\left[a x+\left(b+\alpha_{2}\right) y+(1+c) z+\left(1+\alpha_{1}\right) u\right] \int_{t-r(t)}^{t} \\
& {\left[f_{x}(\cdot) y(s)+f_{y}(\cdot) z(s)+f_{z}(\cdot) u(s)\right.} \\
& \left.+g_{x}(x, y) y(s)+g_{y}(x, y) z(s)+h^{\prime}(x(s)) y(s)\right] d s,
\end{aligned}
$$

where $\phi(\cdot)=\phi(t, x, y, z, u), f(\cdot)=f(x, y, z), f_{x}(\cdot)=f_{x}(x, y, z), f_{y}(\cdot)=$ $f_{y}(x, y, z)$ and $f_{z}(\cdot)=f_{z}(x, y, z)$. Rearranging equation 3.22 , to get 
$V_{3.2}^{\prime}\left(X_{t}\right)=\frac{1}{4} \sum_{i=1}^{7} W_{i}-\left(1-r^{\prime}(t)\right) \int_{t-r(t)}^{t}\left[\mu_{1} y^{2}(s)+\mu_{2} z^{2}(s)+\mu_{3} u^{2}(s)\right] d s+W_{8}$

$+r(t)\left[\mu_{1} y^{2}+\mu_{2} z^{2}+\mu_{3} u^{2}\right]+y \int_{0}^{y} g_{x}(x, s) d s$,

where

$$
\begin{aligned}
W_{1}:= & a \frac{h(x)}{x} x^{2}+\left[\left(\alpha_{2}+b\right) \frac{g(x, y)}{y}-\left(a b+h^{\prime}(x)\right)\right] y^{2} \\
& +\left[(1+c) \frac{f(\cdot)}{z}-\left(a \alpha_{2}+\left(\alpha_{1}+b\right) c\right)\right] z^{2}+\left[\left(1+\alpha_{1}\right) \phi(\cdot)-(1+c)\right] u^{2} ; \\
W_{2}:= & a \frac{h(x)}{x} x^{2}+4\left[\left(b \frac{h(x)}{x}+a \frac{g(x, y)}{y}\right)-a^{2}\right] x y+\left[\left(\alpha_{2}+b\right) \frac{g(x, y)}{y}-\left(a b+h^{\prime}(x)\right)\right] y^{2} ; \\
W_{3}:= & a \frac{h(x)}{x} x^{2}+4\left[\left(c \frac{h(x)}{x}+a \frac{f(\cdot)}{z}\right)-a b\right] x z+\left[(1+c) \frac{f(\cdot)}{z}-\left(a \alpha_{2}+\left(\alpha_{1}+b\right) c\right)\right] z^{2} ; \\
W_{4}:= & a \frac{h(x)}{x} x^{2}+4\left[\left(\frac{h(x)}{x}+a \phi(\cdot)\right)-a c\right] x u+\left[\left(1+\alpha_{1}\right) \phi(\cdot)-(1+c)\right] u^{2} ; \\
W_{5}:= & {\left[\left(\alpha_{2}+b\right) \frac{g(x, y)}{y}-\left(a b+h^{\prime}(x)\right)\right] y^{2}+4\left[\left(c \frac{g(x, y)}{y}+\left(b+\alpha_{2}\right) \frac{f(\cdot)}{z}\right)\right.} \\
& \left.-\left(\alpha_{1} h^{\prime}(x)+a c+b \alpha_{2}-d \alpha_{1}+b^{2}\right)\right] y z+\left[(1+c) \frac{f(\cdot)}{z}-\left(a \alpha_{2}+\left(\alpha_{1}+b\right) c\right)\right] z^{2} ; \\
W_{6}:= & {\left[\left(\alpha_{2}+b\right) \frac{g(x, y)}{y}-\left(a b+h^{\prime}(x)\right)\right] y^{2}+4\left[\left(\left(1+\alpha_{1}\right) \frac{g(x, y)}{y}+\left(b+\alpha_{2}\right) \phi(\cdot)\right)\right.} \\
& \left.-\left(a\left(1+\alpha_{2}\right)+\left(\alpha_{1}+b\right) c\right)\right] y u+\left[\left(1+\alpha_{1}\right) \phi(\cdot)-(1+c)\right] u^{2} ; \\
& {\left[(1+c) \frac{f(\cdot)}{z}-\left(a \alpha_{2}+\left(\alpha_{1}+b\right) c\right)\right] z^{2}+4\left[\left(\left(1+\alpha_{1}\right) \frac{f(\cdot)}{z}+(1+c) \phi(\cdot)\right)\right.} \\
& \left.-\left(a+b\left(1+\alpha_{1}\right)+c^{2}\right)\right] z u+\left[\left(1+\alpha_{1}\right) \phi(\cdot)-(1+c)\right] u^{2} ;
\end{aligned}
$$

and

$$
\begin{aligned}
W_{8}:= & {\left[a x+\left(b+\alpha_{2}\right) y+(1+c) z+\left(1+\alpha_{1}\right) u\right] \int_{t-r(t)}^{t} } \\
& {\left[f_{x}(\cdot) y(s)+f_{y}(\cdot) z(s)+f_{z}(\cdot) u(s)+g_{x}(x, y) y(s)\right.} \\
& \left.+h^{\prime}(x(s)) y(s)\right] d s .
\end{aligned}
$$


The assumptions of Theorem $3.1 \phi(\cdot) \geq a$ for all $t \geq 0, x, y, z, u, \frac{f(\cdot)}{z} \geq b$ for all $x, y, z \neq 0, \frac{g(x, y)}{y} \geq c$ for all $x, y \neq 0, \frac{h(x)}{x} \geq \delta_{0}$ for all $x \neq 0$ and $h^{\prime}(x) \leq d$ for all $x$ so that

$W_{1} \geq a \delta_{0} x^{2}+\left(a \alpha_{2}(c-a) b-d\right) y^{2}+\left(b-a \alpha_{2}-c \alpha_{1}\right) z^{2}+\left(a\left(1+\alpha_{1}\right)-(1+c)\right) u^{2}$

for all $x, y, z$ and $u$. Equation 3.4 and estimate $3.5 \mathrm{~b}$ result to $\alpha_{2}=\epsilon+\frac{d}{c}$ and $\epsilon=\frac{2 a b}{c}$ respectively so that

$$
a \alpha_{2}(c-a) b-d=(a+c) b .
$$

Also, estimates 3.11 and $3.5 \mathrm{~b}$ yield

$$
b-a \alpha_{2}-c \alpha_{1} \geq \frac{\delta}{2 a c} .
$$

Furthermore, since $\alpha_{1}=\epsilon+\frac{1}{a}$ and $\epsilon=\frac{2 c}{a}$ then

$$
a\left(1+\alpha_{1}\right)-(1+c)=a+c .
$$

Using estimates $3.25,3.26$ and 3.27 in estimate 3.24 to get

$$
W_{1} \geq a \delta_{0} x^{2}+(a+c) b y^{2}+\frac{\delta}{2 a c} z^{2}+(a+c) u^{2}
$$

for all $x, y, z$ and $u$. Next, since $W_{2}=W_{2}(x, y), W_{3}=W_{3}(x, z), \cdots, W_{7}=$ $W_{7}(z, u)$ are quadratic functions of two variables, one can generally form a $2 \times 2$ symmetric Hessian matrix

$$
A=\left[\begin{array}{ll}
a & b \\
b & c
\end{array}\right]
$$

which is positive definite if and only if $a>0$ and $a c>b^{2}$. Now for $a>0$ in $W_{i},(i=2,3,4,5,6,7)$ are the positive coefficients of $x^{2}, y^{2}, z^{2}$ and $u^{2}$ in estimate 3.28. The discriminant $b^{2}<a c$ yields the following estimates 


$$
\begin{aligned}
& 4\left[\left(b \frac{h(x)}{x}+a \frac{g(x, y)}{y}\right)-a^{2}\right]^{2}<\left[a \frac{h(x)}{x}\right]\left[\left(\alpha_{2}+b\right) \frac{g(x, y)}{y}-\left(a b+h^{\prime}(x)\right)\right], \\
& 4\left[\left(c \frac{h(x)}{x}+a \frac{f(\cdot)}{z}\right)-a b\right]^{2}<\left[a \frac{h(x)}{x}\right]\left[(1+c) \frac{f(\cdot)}{z}-\left(a \alpha_{2}+\left(\alpha_{1}+b\right) c\right)\right], \\
& 4\left[\left(\frac{h(x)}{x}+a \phi(\cdot)\right)-a c\right]^{2}<\left[a \frac{h(x)}{x}\right]\left[\left(1+\alpha_{1}\right) \phi(\cdot)-(1+c)\right], \\
& 4\left[\left(c \frac{g(x, y)}{y}+\left(b+\alpha_{2}\right) \frac{f(\cdot)}{z}\right)-\left(\alpha_{1} h^{\prime}(x)+a c+b \alpha_{2}-d \alpha_{1}+b^{2}\right)\right]^{2}< \\
& {\left[\left(\alpha_{2}+b\right) \frac{g(x, y)}{y}-\left(a b+h^{\prime}(x)\right)\right]\left[(1+c) \frac{f(\cdot)}{z}-\left(a \alpha_{2}+\left(\alpha_{1}+b\right) c\right)\right],} \\
& 4\left[\left(\left(1+\alpha_{1}\right) \frac{g(x, y)}{y}+\left(b+\alpha_{2}\right) \phi(\cdot)\right)-\left(a\left(1+\alpha_{2}\right)+\left(\alpha_{1}+b\right) c\right)\right]^{2} \\
& <\left[\left(\alpha_{2}+b\right) \frac{g(x, y)}{y}-\left(a b+h^{\prime}(x)\right)\right]\left[\left(1+\alpha_{1}\right) \phi(\cdot)-(1+c)\right], \\
& 4\left[\left(\left(1+\alpha_{1}\right) \frac{f(\cdot)}{z}+(1+c) \phi(\cdot)\right)-\left(a+b\left(1+\alpha_{1}\right)+c^{2}\right)\right] \\
& <\left[(1+c) \frac{f(\cdot)}{z}-\left(a \alpha_{2}+\left(\alpha_{1}+b\right) c\right)\right]\left[\left(1+\alpha_{1}\right) \phi(\cdot)-(1+c)\right] .
\end{aligned}
$$

Employing the above inequalities in $W_{i},(i=2,3,4,5,6,7)$ to get

$$
\begin{aligned}
& W_{2} \geq\left[\sqrt{a \frac{h(x)}{x}}|x|-\sqrt{\left(\alpha_{2}+b\right) \frac{g(x, y)}{y}-\left(a b+h^{\prime}(x)\right)}|y|\right]^{2} \geq 0 \text { ofor all } x, y ; \\
& W_{3} \geq\left[\sqrt{a \frac{h(x)}{x}}|x|-\sqrt{(1+c) \frac{f(\cdot)}{z}-\left(a \alpha_{2}+\left(\alpha_{1}+b\right) c\right)}|z|\right]^{2} \geq 0 \text { for all } x, z ; \\
& W_{4} \geq\left[\sqrt{a \frac{h(x)}{x}}|x|-\sqrt{\left(1+\alpha_{1}\right) \phi(\cdot)-(1+c)}|u|\right]^{2} \geq 0 \text { for all } x, u ; \\
& W_{5} \geq\left[\sqrt{\left(\alpha_{2}+b\right) \frac{g(x, y)}{y}-\left(a b+h^{\prime}(x)\right)}|y|-\sqrt{(1+c) \frac{f(\cdot)}{z}-\left(a \alpha_{2}+\left(\alpha_{1}+b\right) c\right)}|z|\right]^{2} \geq 0 \\
& \text { for ally, } z \text {; } \\
& W_{6} \geq\left[\sqrt{\left(\alpha_{2}+b\right) \frac{g(x, y)}{y}-\left(a b+h^{\prime}(x)\right)}|y|-\sqrt{\left(1+\alpha_{1}\right) \phi(\cdot)-(1+c)}|u|\right]^{2} \geq 0 \\
& \text { for ally, } u \text {; } \\
& W_{7} \geq\left[\sqrt{(1+c) \frac{f(\cdot)}{z}-\left(a \alpha_{2}+\left(\alpha_{1}+b\right) c\right)}|z|-\sqrt{\left(1+\alpha_{1}\right) \phi(\cdot)-(1+c)}|u|\right]^{2} \geq 0
\end{aligned}
$$


Next, applying the obvious inequality $2 p q<p^{2}+q^{2}$, the assumptions $\left|f_{x}(\cdot)\right| \leq M_{1},\left|f_{y}(\cdot)\right| \leq M_{2},\left|f_{z}(\cdot)\right| \leq M_{3},\left|g_{x}(x, y)\right| \leq M_{4},\left|g_{y}(x, y)\right| \leq M_{5}$, and $\left|h^{\prime}(x)\right| \leq M_{6}$ for all $x, y, z$, it follows that

$$
\begin{aligned}
& W_{8} \leq \frac{1}{2} r(t) \sum_{i=1}^{6} M_{i}\left[a x^{2}+\left(\alpha_{2}+b\right) y^{2}+(1+c) z^{2}+\left(1+\alpha_{1}\right) u^{2}\right] \\
& +\frac{1}{2}\left(a+b+c+\alpha_{1}+\alpha_{2}+2\right) \int_{t-r(t)}^{t} \\
& {\left[\left(M_{1}+M_{4}+M_{6}\right) y^{2}(s)+\left(M_{2}+M_{5}\right) z^{2}(s)+M_{3} u^{2}(s)\right] d s .}
\end{aligned}
$$

Combining estimates $W_{i}(i=1,2, \cdots, 8)$, noting that $r(t) \leq \gamma, r^{\prime}(t) \leq \beta$ for all $t \geq 0$, and $g_{x}(x, y) \leq 0$ for all $x, y$, then equation 3.23 becomes

$$
\begin{aligned}
& V_{3.2}^{\prime}\left(X_{t}\right) \leq-\frac{a}{4}\left[\delta_{0}-2 \gamma \sum_{i=1}^{6} M_{i}\right] x^{2} \\
& -\frac{1}{4}\left[(a+c) b-\left(2\left(b+\alpha_{2}\right) \sum_{i=1}^{6} M_{i}+4 \mu_{1}\right) \gamma\right] y^{2} \\
& -\frac{1}{4}\left[\frac{\delta}{2 a c}-\left(2(1+c) \sum_{i=1}^{6} M_{i}+4 \mu_{2}\right) \gamma\right] z^{2} \\
& -\frac{1}{4}\left[a+c-\left(2\left(1+\alpha_{1}\right) \sum_{i=1}^{6} M_{i}+4 \mu_{3}\right) \gamma\right] u^{2} \\
& -\left[(1-\beta) \mu_{1}-\frac{1}{2}\left(M_{1}+M_{4}+M_{6}\right)\left(a+b+c+\alpha_{1}+\alpha_{2}+2\right)\right] \int_{t-r(t)}^{t} y^{2}(s) d s \\
& -\left[(1-\beta) \mu_{2}-\frac{1}{2}\left(M_{2}+M_{5}\right)\left(a+b+c+\alpha_{1}+\alpha_{2}+2\right)\right] \int_{t-r(t)}^{t} z^{2}(s) d s \\
& -\left[(1-\beta) \mu_{3}-\frac{1}{2} M_{3}\left(a+b+c+\alpha_{1}+\alpha_{2}+2\right)\right] \int_{t-r(t)}^{t} u^{2}(s) d s .
\end{aligned}
$$

Choose

$$
\begin{gathered}
\mu_{1}=\frac{1}{2}\left(M_{1}+M_{4}+M_{6}\right)\left(a+b+c+\alpha_{1}+\alpha_{2}+2\right)(1-\beta)^{-1}, \\
\mu_{2}=\frac{1}{2}\left(M_{2}+M_{5}\right)\left(a+b+c+\alpha_{1}+\alpha_{2}+2\right)(1-\beta)^{-1}
\end{gathered}
$$

and

$$
\mu_{3}=\frac{1}{2} M_{3}\left(a+b+c+\alpha_{1}+\alpha_{2}+2\right)(1-\beta)^{-1},
$$

the inequality 3.29 becomes 


$$
\begin{aligned}
& V_{3.2}^{\prime}\left(X_{t}\right) \leq-\frac{a}{4}\left[\delta_{0}-2 \gamma \sum_{i=1}^{6} M_{i}\right] x^{2}-\frac{1}{4}[(a+c) b \\
& \left.-\left(2\left(b+\alpha_{2}\right) \sum_{i=1}^{6} M_{i}+2\left(M_{1}+M_{4}+M_{6}\right)\left(a+b+c+\alpha_{1}+\alpha_{2}+2\right)(1-\beta)^{-1}\right) \gamma\right] y^{2} \\
& -\frac{1}{4}\left[\frac{\delta}{2 a c}-\left(2(1+c) \sum_{i=1}^{6} M_{i}+2\left(M_{2}+M_{5}\right)\left(a+b+c+\alpha_{1}+\alpha_{2}+2\right)(1-\beta)^{-1}\right) \gamma\right] z^{2} \\
& -\frac{1}{4}\left[a+c-\left(2\left(1+\alpha_{1}\right) \sum_{i=1}^{6} M_{i}+2 M_{3}\left(a+b+c+\alpha_{1}+\alpha_{2}+2\right)(1-\beta)^{-1}\right) \gamma\right] u^{2} .
\end{aligned}
$$

Inequality 3.30 invoke the existence of a positive constant $\beta_{3}=\beta_{3}\left(a, b, c, \alpha_{1}, \alpha_{2}, \delta, \delta_{0}, \beta, \gamma, M_{i}\right)(i=1,2, \cdots, 6)$ such that

$$
V_{3.2}^{\prime}\left(X_{t}\right) \leq-\beta_{3}\left(x^{2}+y^{2}+z^{2}+u^{2}\right)
$$

for all $t \geq 0, x, y, z$ and $u$, provided that the inequality 3.5a hold. Estimate 3.31 satisfies inequality 3.21 with $\beta_{3}$ equivalent to $D_{3}$. This completes the prove Lemma 3.4.

Proof. [Proof of Theorem 3.1] Let $X_{t}=0$ be the zero solution of system 3.2. Clearly,

$$
V(\mathbf{0})=0
$$

where $\mathbf{0}=(0,0,0,0) \in \mathbf{R}^{4}$. In addition, estimates 3.16 and 3.31 of Lemmas 3.3 and 3.4 imply that, the functional $V\left(X_{t}\right)$ is positive definite and nonincreasing respectively. Thus the trivial solution $X_{t}=0$ is asymptotically stable. This completes the prove of Theorem 3.1.

Proof. $\quad[$ Proof of Theorem 3.2 $]$ Let $X_{t}=0$ be the zero solution of system 3.2. The inequalities 3.7 and 3.21 of Lemmas 3.3 and 3.4 respectively satisfy assumptions of Lemma 2.12, thus by Lemma 2.12 the zero solution, $X_{t}=0$, of system 3.2 is uniformly asymptotically stable. This completes the proof of Theorem 3.2.

\section{Boundedness, Existence and Uniqueness Theorems}

This section presents uniform ultimate boundedness, existence and uniqueness of solutions for system 1.2. The following results abound. 
Theorem 4.1. If in addition to the assumptions of Theorem 3.2,

$|P(\cdot)| \leq \varphi_{1}(t)+\varphi_{2}(t)(|x|+|y|+|z|+|u|+|x(t-r(t))|+|y(t-r(t))|+|z(t-r(t))|)$

where $\varphi_{1}$ and $\varphi_{2}$ are continuous functions of $t$ satisfying

$$
\varphi_{1}(t) \leq \eta, 0<\eta<\infty
$$

and there exists $\epsilon_{0}>0$ such that for all $t \geq 0$

$$
0 \leq \varphi_{2} \leq \epsilon_{0}
$$

Then the solutions $\left(X_{t}\right)$ of system 1.2 are uniformly bounded and uniformly ultimately bounded, provided that the inequality 3.5a holds true.

Proof. Let $\left(X_{t}\right)$ be any solution of the system 1.2. The continuous partial derivatives of the functional $V\left(X_{t}\right)$, defined by equation 3.3 with respect to independent variable $t$ along a solution of the system 1.2 is

$(4.4) V_{1.2}^{\prime}\left(X_{t}\right)=V_{3.2}^{\prime}\left(X_{t}\right)+\left(a x+\left(b+\alpha_{2}\right) y+(1+c) z+\left(1+\alpha_{1}\right) u\right) P(\cdot)$,

where $V_{3.2}^{\prime}\left(X_{t}\right)$ is defined by 3.22 . The inequality 3.31 and the fact that $x<|x|$ for all real $x$, then equation 4.4 gives rise to the existence of a positive constant $\beta_{4}=\beta_{4}\left(a, b, c, \alpha_{1}, \alpha_{2}\right)$ such that

$(4.5) V_{1.2}^{\prime}\left(X_{t}\right) \leq-\beta_{3}\left(x^{2}+y^{2}+z^{2}+u^{2}\right)+\beta_{4}(|x|+|y|+|z|+|u|)|P(\cdot)|$,

where $\beta_{4}:=\max \left\{a, b+\alpha, 1+c, 1+\alpha_{1}\right\}$. Using inequalities 4.1, 4.2, 4.3 in estimate 4.5 and choose $\epsilon_{0}<8^{-1} \beta_{3} \beta_{4}^{-1}$ there exist positive constants $\beta_{5}=\beta_{5}\left(\beta_{3}, \beta_{4}, \epsilon_{0}\right)$ and $\beta_{6}=\beta_{6}\left(\beta_{4}, \eta\right)$ such that

$$
V_{1.2}^{\prime}\left(X_{t}\right) \leq-\beta_{5}\left(x^{2}+y^{2}+z^{2}+u^{2}\right)+\beta_{6}(|x|+|y|+|z|+|u|)
$$

for all $t \geq 0, x, y, z$ and $u$, where $\beta_{5}:=\beta_{3}-\beta_{4} \epsilon_{0}$ and $\beta_{6}:=\eta \beta_{4}$. Estimate 4.6 can be recast in the form

$$
V_{1.2}^{\prime}\left(X_{t}\right) \leq-\beta_{7}\left(x^{2}+y^{2}+z^{2}+u^{2}\right)
$$

for all $t \geq 0, x, y, z, u$ provided that

$$
\left(x^{2}+y^{2}+z^{2}+u^{2}\right)^{1 / 2} \geq \beta_{8}
$$


where $\beta_{7}:=\frac{1}{2} \beta_{5}$ and $\beta_{8}:=2^{3 / 2} \beta_{5}^{-1} \beta_{6}$. Inequalities $3.16,3.20$, estimates 3.19 and 4.7, the assumptions of Lemma 2.13 are satisfied, hence by Lemma 2.13 all solutions of system 1.2 are uniformly bounded and uniformly ultimately bounded. This completes the prove of Theorem 4.1.

Theorem 4.2. If assumptions of Theorem 3.2 hold, then there exists a unique $\omega$-periodic solution for system 1.2.

Proof. Let $\left(X_{t}\right)$ be any solution of system 1.2. In view of the inequalities $3.16,3.20$, estimates $3.17,3.18,3.19$ and inequality 4.7 , assumptions of Lemmas 2.10 and 2.11 hold, thus by Lemmas 2.10 and 2.11 there exists a unique $\omega$-periodic solution for system 1.2. This completes the prove of Theorem 4.2. $\square$ Next, if the forcing term in equation 1.1 and system 1.2 is replaced by $P(t)$ with $P \in \mathbf{R}^{+}$, then equation 1.1 and system 1.2 specialize to

$x^{(4)}(t)+\phi\left(t, x, x^{\prime}, x^{\prime \prime}, x^{\prime \prime \prime}\right) x^{\prime \prime \prime}+f(\cdot)+g\left(x(t-r(t)), x^{\prime}(t-r(t))\right)+h(x(t-r(t)))=P(t)$

and

$$
\begin{aligned}
x^{\prime}(t) & =y(t), y^{\prime}(t)=z(t), z^{\prime}(t)=u(t), u^{\prime}(t) \\
& =P(t)-\phi(t, x, y, z, u) u-f(\cdot)-g(x, y) \\
& -h(x)+\int_{t-r(t)}^{t}\left[f_{x}(\cdot) y(s)+f_{y}(\cdot) z(s)+f_{z}(\cdot) u(s)+g_{x}(\cdot) y(s)\right. \\
& \left.+g_{y}(\cdot) z(s)+h^{\prime}(x(s)) y(s)\right] d s
\end{aligned}
$$

where the functions $\phi, f, g, h$ are defined in Section 1 . The following results are presented.

Corollary 4.3. Suppose that assumptions of Theorem 3.2 hold true, in addition, if the function $P$ in 4.9 is bounded by a finite constant. Then the solution $\left(X_{t}\right)$ of 4.9 is uniformly bounded and uniformly ultimately bounded.

Proof. The prove of this corollary is similar to the prove of Theorem 4.1, hence it is omitted. This completes the prove of Corollary 4.3.

Corollary 4.4. If the assumptions of Corollary 4.3 hold, then there exists a unique $\omega$-periodic solution for system 4.9. 
Proof. The prove of Corollary 4.4 is similar to the prove of Theorem 4.2 , hence it is omitted. This completes the prove of Corollary 4.4.

\section{Examples and Discussions}

In this section two examples will be presented to illustrate the authenticity of the obtained theoretical results of Sections 3 and 4.

Example 5.1. Consider the following fourth order nonlinear functional differential equation

$$
\begin{aligned}
& x^{(4)}+\left[2+t+\left(x+x^{\prime}\right)^{2}+\left(x^{\prime \prime}+x^{\prime \prime \prime}\right)^{2}\right] x^{\prime \prime \prime} \\
& +\left[6+\frac{1}{2+|x(t-r(t))|+\left|x^{\prime}(t-r(t))\right|+\left|x^{\prime \prime}(t-r(t))\right|}\right] x^{\prime \prime}(t-r(t)) \\
& +\left[1+\frac{1}{1+\left|x(t-r(t)) x^{\prime}(t-r(t))\right|+x^{\prime 2}(t-r(t))}\right] x^{\prime}(t-r(t)) \\
& +\left[1+\frac{1}{1+x(t-r(t))}\right] x(t-r(t))=0 .
\end{aligned}
$$

Equation 5.1 in its equivalent system of first order differential equations

$$
\begin{aligned}
& x^{\prime}=y, y^{\prime}=z, z^{\prime}=u, \\
& u^{\prime}=-\left[2+t+(x+y)^{2}+(z+u)^{2}\right] u-\left[6+\frac{1}{2+|x|+|y|+|z|}\right] z-\left[1+\frac{1}{1+|x y|+y^{2}}\right] y \\
& -\left[1+\frac{1}{1+x^{2}}\right] x-\int_{t-r(t)}^{t} \frac{z(s)}{(2+|x(s)|+|y(s)|+|z(s)|)^{2}} y(s) d s \\
& -\int_{t-r(t)}^{t} \frac{z(s)}{(2+|x(s)|+|y(s)|+|z(s)|)^{2}} z(s) d s-\int_{t-r(t)}^{t}\left[\frac{y^{2}(s)}{\left(1+|x(s) y(s)|+y^{2}(s)\right)^{2}}\right] y(s) d s \\
& +\int_{t-r(t)}^{t}\left[6+\frac{1}{2+|x(s)|+|y(s)|}-\frac{z(s)}{(2+|x(s)|+|y(s)|)^{2}}\right] u(s) d s \\
& +\int_{t-r(t)}^{t}\left[1+\frac{1}{1+|x(s) y(s)|+y^{2}(s)}-\frac{y(s)(x(s)+y(s))}{\left(1+|x(s) y(s)|+y^{2}(s)\right)^{2}}\right] z(s) d s \\
& +\int_{t-r(t)}^{t}\left[1+\frac{1}{1+x^{2}(s)}-\frac{2 x^{2}(s)}{\left(1+x^{2}(s)\right)^{2}}\right] y(s) d s .
\end{aligned}
$$


Next, comparing systems 3.2 and 5.2, the following equivalence are noticed:

(i) The function

$$
\phi(t, x, y, z, u) \equiv 2+t+(x+y)^{2}+(z+u)^{2} .
$$

Now since

$$
\phi(\mathbf{0})=2=\phi(0, x=-y, z=-u)
$$

where $\mathbf{0}=(0,0,0,0,0)$ and the iterative limits of the function $\phi$ with respect to $t, x, y, z$ and $u$ is

$$
\begin{aligned}
& \lim _{t \rightarrow \infty}\left(\lim _{x \rightarrow \infty}\left(\lim _{y \rightarrow \infty}\left(\lim _{z \rightarrow \infty}\left(\lim _{u \rightarrow \infty} \phi(t, x, y, z, u)\right)\right)\right)\right) \\
& =\lim _{x \rightarrow \infty}\left(\lim _{y \rightarrow \infty}\left(\lim _{z \rightarrow \infty}\left(\lim _{u \rightarrow \infty}\left(\lim _{t \rightarrow \infty} \phi(t, x, y, z, u)\right)\right)\right)\right) \\
& =\lim _{y \rightarrow \infty}\left(\lim _{z \rightarrow \infty}\left(\lim _{u \rightarrow \infty}\left(\lim _{t \rightarrow \infty}\left(\lim _{x \rightarrow \infty} \phi(t, x, y, z, u)\right)\right)\right)\right) \\
& \left.\left.\left.\left.=\lim _{z \rightarrow \infty}\left(\lim _{u \rightarrow \infty}\left(\lim _{t \rightarrow \infty}\left(\lim _{x \rightarrow \infty}\left(\lim _{y \rightarrow \infty} \phi(t, x, y, z, u)\right)\right)\right)\right)\right\} \lim _{z \rightarrow \infty} \phi(t, x, y, z, u)\right)\right)\right) \\
& =\lim _{u \rightarrow \infty}\left(\operatorname { l i m } _ { t \rightarrow \infty } \left(\operatorname { l i m } _ { x \rightarrow \infty } \left(\lim _{y \rightarrow \infty}\left(\lim _{z}\right)\right.\right.\right. \\
& =\infty .
\end{aligned}
$$

It follows that the function $\phi$ is bounded below by 2 and is unbounded above, this is

$$
2=a \leq \phi(t, x, y, z, u)<\infty
$$

for all $t \geq 0, x, y, z$ and $u$.

(ii) The function

$$
f(x, y, z) \equiv 6 z+\frac{z}{2+|x|+|y|+|z|} .
$$

Define

$$
F(x, y, z)=\frac{1}{2+|x|+|y|+|z|},
$$

since $2+|x|+|y|+|z| \geq 2$ for all $x, y, z$, and $F(0,0,0)=\frac{1}{2}$, it follows that $F$ is a decreasing function for all $x, y, z$ and its iterative limits with respect to variables $x, y$ and $z$ is 


$$
\begin{aligned}
\lim _{x \rightarrow \infty} & \left(\lim _{y \rightarrow \infty}\left(\lim _{z \rightarrow \infty} F(x, y, z)\right)\right) \\
& =\lim _{y \rightarrow \infty}\left(\lim _{z \rightarrow \infty}\left(\lim _{x \rightarrow \infty} F(x, y, z)\right)\right) \\
& =\lim _{z \rightarrow \infty}\left(\lim _{x \rightarrow \infty}\left(\lim _{y \rightarrow \infty} F(x, y, z)\right)\right) \\
& =0
\end{aligned}
$$

it follows that

$$
0 \leq F(x, y, z) \leq \frac{1}{2}
$$

for all $x, y, z$.

$$
6=b \leq \frac{f(x, y, z)}{z}
$$

for all $x, y, z$. Moreover, the first partial derivative of the function $f$ with respect to variable $x>0$ is

$$
f_{x}(x, y, z)=-\frac{z}{(2+|x|+|y|+|z|)^{2}} .
$$

Since the iterative limits of $f_{x}(x, y, z)$ is

$$
\begin{aligned}
\lim _{x \rightarrow \infty} & \left(\lim _{y \rightarrow \infty}\left(\lim _{z \rightarrow \infty}\left|f_{x}(x, y, z)\right|\right)\right) \\
& =\lim _{y \rightarrow \infty}\left(\lim _{z \rightarrow \infty}\left(\lim _{x \rightarrow \infty}\left|f_{x}(x, y, z)\right|\right)\right) \\
& =\lim _{z \rightarrow \infty}\left(\lim _{x \rightarrow \infty}\left(\lim _{y \rightarrow \infty}\left|f_{x}(x, y, z)\right|\right)\right) \\
& =0
\end{aligned}
$$

and

$$
\frac{|z|}{(2+|x|+|y|+|z|)^{2}} \leq 1
$$

for all $x, y, z$ it follows that

$$
\left|f_{x}(x, y, z)\right| \leq M_{1}=1
$$

for all $x, y$ and $z$. Furthermore, the first partial derivative of $f$ with respect to variable $y>0$ is

$$
f_{y}(x, y, z)=-\frac{z}{(2+|x|+|y|+|z|)^{2}},
$$


since the iterative limits of $\left|f_{y}(x, y, z)\right|$ is

$$
\begin{aligned}
\lim _{x \rightarrow \infty} & \left(\lim _{y \rightarrow \infty}\left(\lim _{z \rightarrow \infty}\left|f_{y}(x, y, z)\right|\right)\right) \\
& =\lim _{y \rightarrow \infty}\left(\lim _{z \rightarrow \infty}\left(\lim _{x \rightarrow \infty}\left|f_{y}(x, y, z)\right|\right)\right) \\
& =\lim _{z \rightarrow \infty}\left(\lim _{x \rightarrow \infty}\left(\lim _{y \rightarrow \infty}\left|f_{y}(x, y, z)\right|\right)\right) \\
& =0
\end{aligned}
$$

and

$$
\frac{|z|}{(2+|x|+|y|+|z|)^{2}} \leq 1
$$

for all $x, y, z$ it follows that

$$
\left|f_{y}(x, y, z)\right| \leq M_{2}=1
$$

for all $x, y$ and $z$. What is more, the first partial derivative of $f$ with respect to variable $z>0$ is

$$
f_{z}(x, y, z)=6+\frac{1}{2+|x|+|y|+|z|}-\frac{z}{(2+|x|+|y|+|z|)^{2}} .
$$

It is that $f_{z}(0,0,0)=6.5$ The iterative limits of $f_{z}$ with respect to variables $x, y$ and $z$ is

$$
\begin{aligned}
\lim _{x \rightarrow \infty} & \left(\lim _{y \rightarrow \infty}\left(\lim _{z \rightarrow \infty}\left|f_{z}(x, y, z)\right|\right)\right) \\
& =\lim _{y \rightarrow \infty}\left(\lim _{z \rightarrow \infty}\left(\lim _{x \rightarrow \infty}\left|f_{z}(x, y, z)\right|\right)\right) \\
& =\lim _{z \rightarrow \infty}\left(\lim _{x \rightarrow \infty}\left(\lim _{y \rightarrow \infty}\left|f_{z}(x, y, z)\right|\right)\right) \\
& =6
\end{aligned}
$$

it follows that

$$
f_{z}(x, y, z) \leq M_{3}=6.5
$$

for all $x, y, z$.

(iii) The function

$$
g(x, y) \equiv y+\frac{y}{1+|x y|+y^{2}}
$$


Let

$$
G(x, y):=\frac{1}{1+|x y|+y^{2}},
$$

since $1+|x y|+y^{2} \geq 1$ for all $x, y, G(0,0)=1$ and the iterative limits of $G(x, y)$ is

$$
\lim _{x \rightarrow \infty}\left(\lim _{y \rightarrow \infty} G(x, y)\right)=\lim _{y \rightarrow \infty}\left(\lim _{x \rightarrow \infty} G(x, y)\right)=0
$$

it follows that $G(x, y)$ is a decreasing function and that

$$
0 \leq G(x, y) \leq 1
$$

for all $x$ and $y$. Thus

$$
1=c \leq \frac{g(x, y)}{y} \leq c_{1}=2
$$

for all $x$ and $y \neq 0$. In addition, the first partial derivative of $g$ with respect to $x>0$ is

$$
g_{x}(x, y)=-\frac{y^{2}}{\left(1+|x y|+y^{2}\right)^{2}} \leq 0
$$

for all $x$ and $y$. Furthermore, since

$$
\frac{y^{2}}{\left(1+|x y|+y^{2}\right)^{2}}<1
$$

for all $x, y$,

$$
\lim _{x \rightarrow \infty}\left(\lim _{y \rightarrow \infty}\left|g_{x}(x, y)\right|\right)=\lim _{y \rightarrow \infty}\left(\lim _{x \rightarrow \infty}\left|g_{x}(x, y)\right|\right)=0
$$

and also Figure 5.1 implies that 


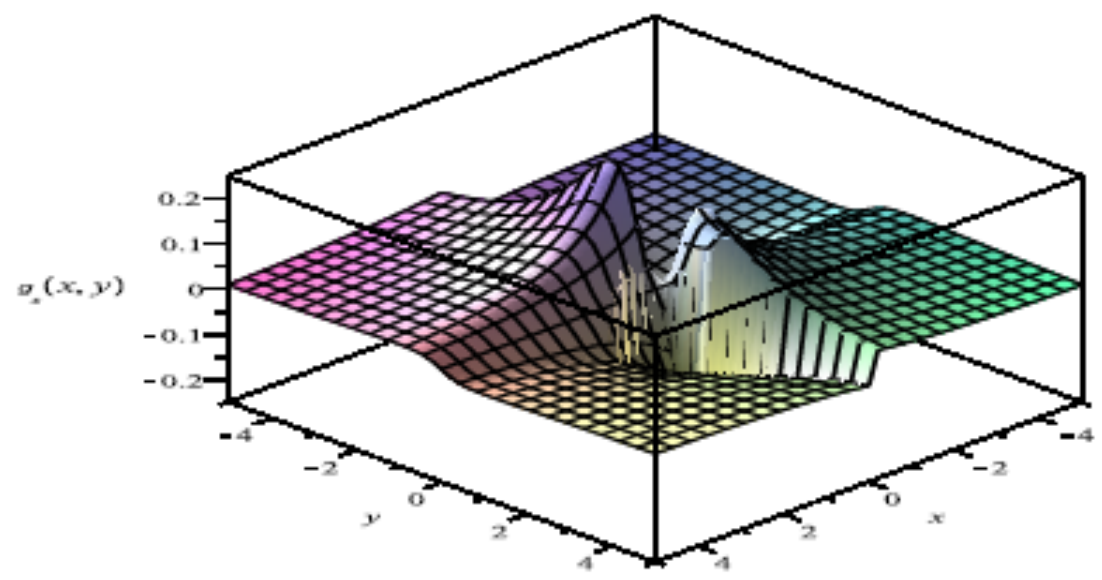

Figure 5.1: The behaviour of $\left|g_{x}(x, y)\right|$ for all $(x, y) \in[-4,4]$

$$
\left|g_{x}(x, y)\right| \leq M_{4}=1
$$

for all $x$ and $y$. Moreover,

$$
\left|g_{y}(x, y)\right| \leq 1+\frac{1}{1+|x y|+y^{2}}+\frac{|y|(|x|+2|y|)}{\left(1+|x y|+y^{2}\right)^{2}} \leq M_{5}=2
$$

for all $x, y$ see Figure 5.2. 


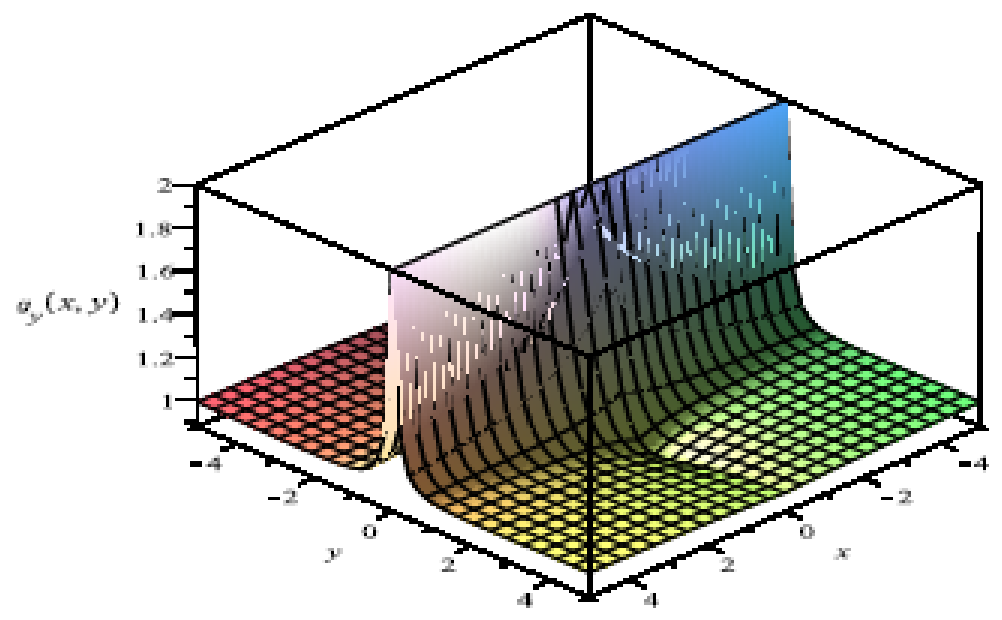

Figure 5.2: The behaviour of $\left|g_{y}(x, y)\right|$ for all $(x, y) \in[-4,4]$

(iv) The function

$$
h(x) \equiv x+\frac{x}{1+x^{2}} .
$$

Define

$$
H(x)=\frac{1}{1+x^{2}},
$$

since $H(0)=1$ and $\lim _{x \rightarrow \infty} H(x)=0$, to conclude that

$$
0 \leq H(x) \leq 1
$$

for all $x$. These inequalities, imply that

$$
1=\delta_{0} \leq \frac{h(x)}{x} \leq d_{1}=2
$$

for all $x \neq 0$. The derivative of $h$ with respect to $x$ is

$$
h^{\prime}(x)=1+\frac{1}{1+x^{2}}-\frac{2 x^{2}}{\left(1+x^{2}\right)^{2}} \leq 1+\frac{1}{1+x^{2}} \leq d=2
$$

and

$$
\left|h^{\prime}(x)\right| \leq M_{6}=2,
$$

for all $x$, see Figure 5.3 


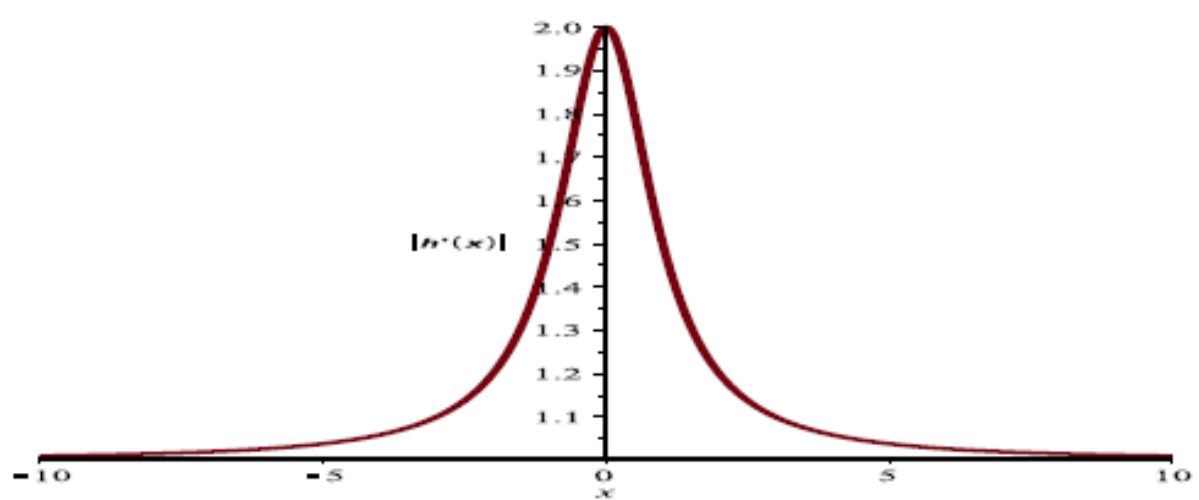

Figure 5.3: The behaviour of $\left|h^{\prime}(x)\right|$ for all $x \in[-10,10]$.

(v) The inequalities in items (v) of Theorem 3.1 are

$$
a b-c=11>0 \text { and } a b c-c^{2}-a^{2} d=3 \geq \delta,
$$

here $\delta=2.999$ is chosen. The following constants are also calculated: $\alpha_{0}=15$, the constant $\epsilon$ is such that

$$
0.017 \leq \epsilon \leq 24=\max \{1,24\}
$$

and $\epsilon=0.0171$ is chosen. Also, $\alpha_{1}=0.5171, \alpha_{2}=2.0171, A_{1}=27$, $A_{2}=224.46, A_{3}=240, A_{4}=216.9 ;$ and

(vi) The constant $\gamma$ is such that

$$
\gamma<0.013=\min \{0.037,0.027,0.013,0.014\} .
$$

Items (i) to (vi) above fulfill the assumptions of Theorems 3.1 and 3.2, hence by Theorems 3.1 and 3.2 the zero solution of equation 3.2 is not only asymptotically stable, but also uniformly asymptotically stable. 
Example 5.2. Consider the following fourth order nonlinear functional differential equation

$$
\begin{aligned}
& x^{(4)}+\left[2+t+\left(x+x^{\prime}\right)^{2}+\left(x^{\prime \prime}+x^{\prime \prime \prime}\right)^{2}\right] x^{\prime \prime \prime} \\
& +\left[6+\frac{1}{\left.2+|x(t-r(t))|+\left|x^{\prime}(t-r(t))\right|+\mid x^{\prime \prime}(t-r(t))\right)}\right] x^{\prime \prime}(t-r(t)) \\
& +\left[1+\frac{1}{1+\left|x(t-r(t)) x^{\prime}(t-r(t))\right|+x^{\prime 2}(t-r(t))}\right] x^{\prime}(t-r(t)) \\
& +\left[1+\frac{1}{1+x(t-r(t))}\right] x(t-r(t))=P(\cdot)
\end{aligned}
$$

where

$P(\cdot):=\frac{1}{1+2 t}+\frac{1}{\left(10+t^{2}\right)\left(\left|x x^{\prime}\right|+\left|x^{\prime \prime} x^{\prime \prime \prime}\right|+\left|x(t-r(t)) x^{\prime}(t-r(t))\right|+x^{\prime \prime 2}(t-r(t))\right)}$

Equation 5.3 in its equivalent system of first order differential equations

$$
\begin{aligned}
& x^{\prime}=y, y^{\prime}=z, z^{\prime}=u \\
& u^{\prime}=\frac{1}{1+2 t}+\frac{1}{\left(10+t^{2}\right)\left(|x y|+|z u|+|x(t-r(t)) y(t-r(t))|+z^{2}(t-r(t))\right)} \\
& -\left[2+t+(x+y)^{2}+(z+u)^{2}\right] u-\left[6+\frac{1}{2+|x|+|y|+|z|}\right] z \\
& -\left[1+\frac{1}{1+|x y|+y^{2}}\right] y-\left[1+\frac{1}{1+x^{2}}\right] x-\int_{t-r(t)}^{t} \frac{z(s)}{(2+|x(s)|+|y(s)|+|z(s)|)^{2}} y(s) d s \\
& -\int_{t-r(t)}^{t} \frac{z(s)}{(2+|x(s)|+|y(s)|+|z(s)|)^{2}} z(s) d s-\int_{t-r(t)}^{t}\left[\frac{y^{2}(s)}{\left(1+|x(s) y(s)|+y^{2}(s)\right)^{2}}\right] y(s) d s \\
& +\int_{t-r(t)}^{t}\left[6+\frac{1}{2+|x(s)|+|y(s)|}-\frac{z(s)}{(2+|x(s)|+|y(s)|)^{2}}\right] u(s) d s \\
& +\int_{t-r(t)}^{t}\left[1+\frac{1}{1+|x(s) y(s)|+y^{2}(s)}-\frac{y(s)(x(s)+y(s))}{\left(1+|x(s) y(s)|+y^{2}(s)\right)^{2}}\right] z(s) d s \\
& +\int_{t-r(t)}^{t}\left[1+\frac{1}{1+x^{2}(s)}-\frac{2 x^{2}(s)}{\left(1+x^{2}(s)\right)^{2}}\right] y(s) d s .
\end{aligned}
$$

Next, comparing systems 1.2 and 5.4, to find that items (i) to (vi) of Example 5.1 hold. In addition

(vii) the function

$$
P(\cdot) \equiv \frac{1}{1+2 t}+\frac{1}{\left(10+t^{2}\right)\left(|x y|+|z u|+|x(t-r(t)) y(t-r(t))|+z^{2}(t-r(t))\right)},
$$


where

$$
\varphi_{1}(t)=\frac{1}{1+2 t} \operatorname{and} \varphi_{2}(t)=\frac{1}{10+t^{2}} .
$$

Since $\varphi_{1}(0)=1,1+2 t>0$ for all $t \geq 0$, then the function $\varphi_{1}(t)$ is decreasing and $\lim _{t \rightarrow \infty} \varphi_{1}(t)=0$ to conclude that

$$
\varphi_{1}(t) \leq \eta=1<\infty
$$

for all $t \geq 0$. Similarly,

$$
0 \leq \varphi_{2}(t) \leq \epsilon_{0}=\frac{1}{10}
$$

for all $t \geq 0$.

Items (i) to (vii) of Examples 5.1 and 5.2 fulfilled assumptions of Theorems 4.1 and 4.2 , hence by Theorems 4.1 and 4.2 solutions $\left(X_{t}\right)$ of 5.4 are uniformly bounded, uniformly ultimately bounded and there exists a unique periodic solution of period $\omega$.

\section{References}

[1] A. T. Ademola, "Periodicity, stability, and boundedness of solutions to certain fourth order delay differential equations", International journal of nonlinear science, vol. 28, no. 1, pp. 20-39, 2019. [On line]. Available: https:// bit.ly/2YwpD06

[2] A. T. Ademola and M. O. Ogundiran, "On the existence and uniqueness of solutions of a generalized Lipschitz ordinary differential equations", Ife journal of science, vol. 9, no. 2, pp. 241-246, 2007, doi: $10.4314 /$ ijs.v9i2.32255

[3] O. A. Adesina and B. S. Ogundare, "Some new stability and boundedness results on a certain fourth order nonlinear differential equation", Nonlinear studies, vol. 19, no. 3, pp. 359-369, 2012. [On line]. Available: https:// bit.ly/ 3oETrlM

[4] S. Balamuralitharan, "Periodic solutions of fourth order delay differential equation", Bulletin of the Iranian Mathematical Society, vol. 41, no, 2, pp. 307-314, 2015. [On line]. Available: https:// bit.ly/3oBAO1 W 
[5] T. A. Burton, Stability and periodic solutions of ordinary and functional differential equations. Orlando, FL: Academic Press, 1985.

[6] T. A. Burton, Volterra integral and differential equations. New York, NY: Academic Press, 1983.

[7] M. Cai and F. Meng, "Stability and boundedness of solutions for a certain fourth order delay differential equation", Annals of applied mathematics, vol. 34, no. 4, pp. 345-357, 2018. [On line]. Available: https:// bit.ly/ 3 jfZ3C9

[8] R. D. Driver, Ordinary and delay differential equations. New York, NY: Springer, 1977, doi: 10.1007/978-1-4684-9467-9

[9] J. K. Hale, Theory of functional differential equations. New York, NY: Springer, 1977, doi: 10.1007/978-1-4612-9892-2

[10] H. Kang and L. Si, "Stability of solutions to certain fourth order delay differential equations", Annals of differential equations, no. 4, pp. 407-413, 2010. [On line]. Available: https:/ / bit.ly/ 3aoNapl

[11] E. Korkmaz and C. Tunç, "Boundedness and square integrability of solutions of nonlinear fourth order differential equations with bounded delay", Electronic journal of differential equations, vol. 2017, Art ID. 47, 2017. [On line]. Available: https:/ / bit.ly/3pzSexm

[12] E. Korkmaz, "Stability and boundedness of solutions of nonlinear fourth order differential equations with bounded delay", Sakarya Üniversitesi Fen Bilimleri Enstitüsü Dergisi, vol. 21, no. 6, pp. 1317-1324, 2017, doi: 10.16984/ saufenbilder.308097

[13] E. Korkmaz, "Stability and square integrability of derivatives of solutions of nonlinear fourth order differential equations with delay", Journal of inequalities and applications, vol. 2017, pp. Art ID. 134, 2017, doi: 10.1186/s13660-017-1353-8

[14] V. Lakshmikantham, L. Wen, and B. Zhang, Theory of differential equations with unbounded delay. Dordrecht: Kluwer, 1994, doi: $10.1007 / 978-1-4615-2606-3$ 
[15] E. O. Okoronkwo, "On stability and boundedness of solutions of a certain fourth order delay differential equation", International journal of mathematics and mathematical sciences, vol. 12, Art ID. 607032, pp. 589-602, 1989, doi: 10.1155/S0161171289000724

[16] M. Rahmane and M. Remili, "On stability and boundedness of solutions of certain non autonomous fourth order delay differential equations", Acta Universitatis Matthiae Belii. Series mathematics (Online), vol. 23, pp. 101-114, 2015. [On line]. Available: https:// bit.ly/2Mdxttb

[17] A. I. Sadek, "On the stability of solutions of certain fourth order delay differential equation", Applied mathematics and computation, vol. 148, no. 2, pp.587-597, 2004, doi: 10.1016/S0096-3003(02)00925-6

[18] A. S. C. Sinha, "On stability of solutions of some third and fourth order delay differential equations", Information and control, vol. 23, no. 2, pp. 165-172, 1973, doi: 10.1016/S0019-9958(73)90651-7

[19] H. O. Te jumola and B. Tchegnani, "Stability, boundedness and existence of periodic solutions of some third order and fourth-order nonlinear delay differential equations", Journal of the Nigerian Mathematical Society, vol. 19, pp. 9-19, 2000.

[20] C. Tunç, "A boundedness criterion for fourth order nonlinear ordinary differential equations with delay", International journal of nonlinear science, vol. 6, no. 3, pp. 195-201, 2008. [On line]. Available; https:// bit.ly/3ctxnYX

[21] C. Tunç, "On stability of solutions of certain fourth order delay differential equations", Applied mathematics and mechanics, vol. 27, pp. 1141-1148, 2006, doi: 10.1007/s10483-006-0815-y

[22] C. Tunç, "On the uniform boundedness of solutions of some nonautonomous differential equations of the fourth order", Applied mathematics and mechanics, vol. 20, pp. 622-628, 1999, doi: 10.1007/ BF02464934

[23] T. Yoshizawa, Stability theory by Liapunov's second method. Tokyo: The Mathematical Society of Japan, 1966.

[24] T. Yoshizawa, Stability theory and existence of periodic solutions and almost periodic solutions. New York, NY: Springer, 1975, doi: $10.1007 / 978-1-4612-6376-0$ 